\title{
Physical parameters along the boundaries of a mid-latitude streamer and in its adjacent regions
}

\author{
R. Susino ${ }^{1}$, R. Ventura ${ }^{2}$, D. Spadaro ${ }^{2}$, A. Vourlidas ${ }^{3}$, and E. Landi ${ }^{3}$ \\ 1 Dipartimento di Fisica e Astronomia - Sezione Astrofisica, Università di Catania, via S. Sofia 78, 95123 Catania, Italy \\ e-mail: sur@oact.inaf.it \\ 2 INAF-Osservatorio Astrofisico di Catania, via S. Sofia 78, 95123 Catania, Italy \\ e-mail: [rve; dspadaro]@oact.inaf.it \\ 3 Naval Research Laboratory, 4555 Overlook Avenue, SW, Washington, DC 20375-5320, USA \\ e-mail: [vourlidas; landi]@nrl.navy.mil
}

Received 4 March 2008 / Accepted 28 May 2008

\begin{abstract}
Context. Coronal streamers appear to be strictly associated with the generation of the slow solar wind, even if a firm identification of the sources of the particle flux within these structures is still an unresolved issue.

Aims. The purpose of this work is to contribute to a better knowledge of the physical characteristics of streamers and of their surroundings in a wide range of heliocentric distances and at both high radial and latitudinal resolutions.

Methods. The analysis is based on spectral observations of a narrow, mid-latitude streamer performed with UVCS/SOHO during one week in May 2004: H I Ly $\alpha$ and O VI resonance doublet line intensities and profiles were obtained at different heliocentric distances and latitudes. In addition, white-light polarized brightness images were taken in the same days of observation, through the LASCO/SOHO C2 coronagraph.

Results. The radial variations in electron density and temperature, H I and O VI kinetic temperatures, and outflow velocities were derived from the observed line intensities, profiles, and $\mathrm{O}$ VI line intensity ratios between 1.6 and $5.0 R_{\odot}$, in two regions, 2-3 arcmin wide, located along the boundaries and in a narrow strip (5-10 arcmin) outside the streamer structure. Significantly high kinetic temperatures and outflow velocities were found in the out-of-streamer region above $3.0 R_{\odot}$ for the $\mathrm{O}$ VI ions and, for the first time, $\mathrm{HI}$ atoms, compared to those obtained along the streamer boundaries. Moreover, the O VI kinetic temperatures and velocities turn out much higher than the $\mathrm{HI}$ ones at any heliocentric distance in all the observed regions. A higher anisotropy is also noticed for the $\mathrm{O}$ VI kinetic temperature in the region flanking the streamer.

Conclusions. The slow coronal wind is found to flow with significantly different speeds and kinetic temperatures along the boundaries of the streamer and in the out-of-streamer regions at all heights, above $3.0-3.5 R_{\odot}$. This fact, consistent with previous studies, indicates that two components of slow wind probably form in the observed regions: one originates just above the streamer cusp and flows with velocities a little higher than $100 \mathrm{~km} \mathrm{~s}^{-1}$, while the other flows along the open magnetic field lines flanking the streamer with velocities slightly lower than the slow wind asymptotic heliospheric value of $\sim 400 \mathrm{~km} \mathrm{~s}^{-1}$, around $5.0 R_{\odot}$.
\end{abstract}

Key words. Sun: corona - Sun: solar wind - Sun: UV radiation

\section{Introduction}

Recent observations of the extended solar corona, carried out with the Ultra-Violet Coronagraph Spectrometer (UVCS, Kohl et al. 1995) on board the SOlar and Heliospheric Observatory (SOHO, Domingo et al. 1995), have significantly contributed to the study of the physical properties of coronal structures at different heights and latitudes and to identifying the sources of fast and slow solar winds. In particular, spectroscopic analysis of ultraviolet line emission in coronal streamers has revealed that they are strictly involved in the generation of the slow-speed component of the solar wind (e.g., Kohl et al. 1997; Raymond et al. 1997).

Streamers are bright, large, and long-lived magnetic structures visible both in white and UV light. They appear to be shaped like pointed helmets or a wide fan of radial rays and extend into the interplanetary medium. During solar maximum they ubiquitously fill the solar corona, while during minimum they are located around the solar equator, forming the so-called streamer belt.
Even though many recent works made it evident that a link exists between streamers and the slow solar wind (see, e.g., Noci et al. 1997; Habbal et al. 1997; Woo \& Martin 1997; Wang et al. 1998; Uzzo et al. 2003), it is not yet clear from which regions within these structures the particle flux originates. Outflow (e.g., Habbal et al. 1997; Strachan et al. 2002; Uzzo et al. 2006, 2007) and abundance (e.g., Raymond et al. 1997; Antonucci et al. 2006; Uzzo et al. 2007) measurements have shown that potential sources of the slow solar wind might be the streamer regions where a transition from closed to open magnetic field lines exists, i.e. the legs and stalk structures. Nevertheless, at present, a real identification of the slow wind acceleration regions is still an open question.

This work is based on the spectral data collected by UVCS/SOHO during one week in May 2004, already described in Spadaro et al. (2007, hereafter Paper I) and pertaining to a narrow, mid-latitude quiescent streamer observed during the declining phase of the solar activity cycle 23 .

While only the results of the analysis concerning the central region of the streamer were presented and discussed in Paper I, 
here we focus on characterizing the physical conditions of the plasma located across the boundaries of the streamer and in its surroundings, with a special emphasis on comparing different regions of the structure. As in Paper I, owing to the changing shape and aspect of the streamer along the line of sight over the observation week as a consequence of the solar rotation, the results obtained can be considered as an average representation of the physical status of the plasma inside and immediately outside streamer structures. To deduce the radial variations in the physical plasma parameters, such as the electron density and temperature, the kinetic temperature, and outflow velocity of the emitting ions in these regions, we adopted the same analysis methods as in Paper I. The purpose of the present work is to contribute to better knowledge of the characteristics of streamers and of their surroundings, which appear to be crucial for firmly identifying the sources of the slow solar wind in the framework of the ongoing campaign devoted to the study of the physical properties of these coronal structures (see, e.g., Uzzo et al. 2006, 2007).

\section{Observations and data analysis}

The spectral data described in Paper I and used in the present work were collected during special observations carried out by UVCS/SOHO in 2004 from May 17 to May 23 (see Table 1 of Paper I). The measurements refer to a narrow, mid-latitude quiescent streamer structure located above the southwest limb of the Sun and consist of radial and latitudinal scans of the extended corona from 1.6 to $5.0 R_{\odot}$ in the $215^{\circ}-240^{\circ}$ range of position angle (PA, measured counterclockwise from solar north pole direction); intensities and profiles of both $\mathrm{HI}$ Ly $\alpha$ and O VI resonance doublet lines were collected during the observations (see Sect. 2 of Paper I for more details). The Ly $\alpha$ data were acquired only during a radial scan started on May 22 and completed on May 23, 2004.

In addition, LASCO (Large Angle Spectroscopic COronagraph, Brueckner et al. 1995) pB images, whose inversion provided electron density maps of the region observed in UV, were taken day by day from May 17 to May 22. On May 18 and 19, moreover, intensities and profiles of the OVI resonance doublet lines were also measured by the spectrometer SUMER (Solar Ultraviolet Measurements of Emitted Radiation, Wilhelm et al. 1995) in two adjacent regions on the solar disc close to the southwest limb, just below the observed streamer. These chromospheric spectral data were used to calculate the proper straylight contribution coming from the solar disc to the O VI line profiles observed in corona (see below).

Standard wavelength and radiometric calibration, flat-field correction, image distortion, stray-light contribution removal, subtraction of the background, and instrumental broadening correction were all applied to both the $\mathrm{HI}_{\mathrm{I}} \mathrm{Ly} \alpha$ and O VI doublet line profiles, as described in Sect. 2 of Paper I. The contribution from the interplanetary scattered Ly $\alpha$ line contained in the observed Ly $\alpha$ profile was also removed. Then the spectra were extracted and single Gaussian fits to the line profiles were obtained to determine their intensity and $1 / e$ half width.

To localize the streamer borders, we adopted the $1 / e$ intensity criterion described, for instance, in Abbo \& Antonucci (2002), for each exposure and spectral line examined. For the northern boundary, however, this was possible only for exposures collected from May 21 to May 23 (i.e., in the 2.5-5.0 $R_{\odot}$ range of heliocentric distance), because in the previous days a wide, faint structure was present in the northward region of the streamer, precluding an accurate identification of the border (see Fig. 2 of Paper I). We then extracted the spectra

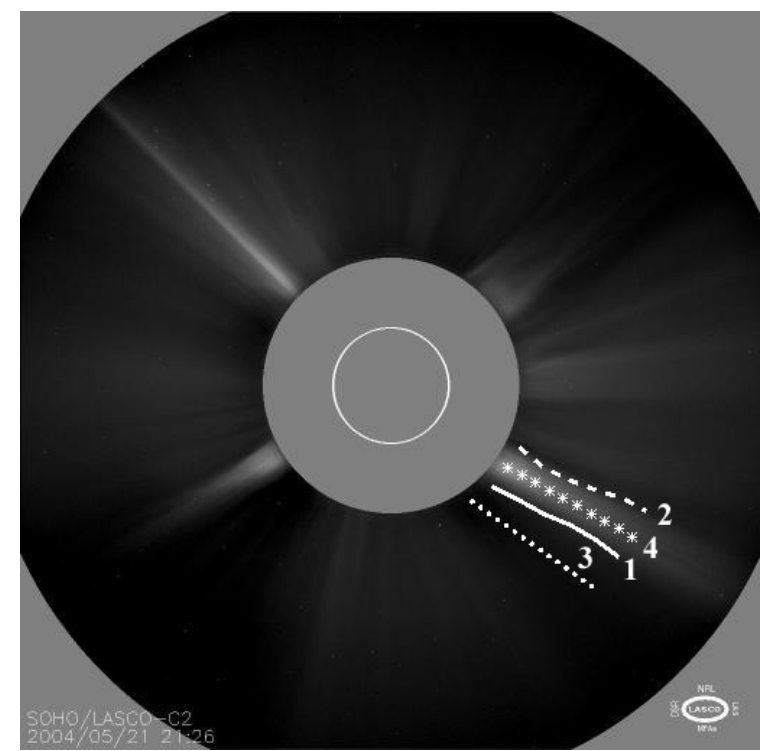

Fig. 1. LASCO C2 white-light image taken on May 21, 2004. The lines mark the positions of the southern (solid line, label 1) and northern (dashed line, label 2) boundaries of the streamer structure and its central region (asterisks, label 4), as deduced from the radial scans performed in that day. The region between the solid and the dotted lines corresponds to the observed out-of-streamer region (label 3).

relevant to two narrow strips centered on the northern and the southern boundaries of the streamer, respectively, and approximately 2-3 arcmin wide for both the H I Ly $\alpha$ and O VI exposures, and a strip, approximately 5-10 arcmin wide, adjacent to the southern border of the structure.

Figure 1 shows a LASCO C2 white-light image of the extended solar corona, obtained on May 21, 2004. The positions of the center of the streamer structure and of its borders, derived as described above, are superimposed on the image. It is also marked the position of the out-of-streamer region adjacent to the southern border of the observed streamer.

To derive the radial profiles of the electron density and temperature, the kinetic temperature, and outflow velocity of the emitting ions along the streamer boundaries and in the considered adjacent region, we made use of the same spectral synthesis code as was adopted in Paper I and iteratively reproduced the observed H I Ly $\alpha$ and O VI spectral emission as closely as possible. As in Paper I, we assumed that the bulk of the coronal emission from the considered region is confined within $\pm 0.2-0.3 R_{\odot}$ from the plane of the sky.

The average disc profiles adopted to synthesize the radiatively excited component of the spectral lines expected from the extended corona are those that gave the best results in the analysis of the central region of the streamer (see Sect. 3.2 of Paper I): i.e. the O VI 1032 and $1037 \AA$ disc intensities listed in Vernazza \& Reeves (1978) and the chromospheric Ly $\alpha$ line profile reported in Gouttebroze et al. (1978).

The atomic data, elemental abundances, and ionization balances used in the synthesis of the O VI and H I Ly $\alpha$ emission are those adopted in the analysis of the central region of the streamer (see Sect. 2 of Paper I and the discussion presented there). 


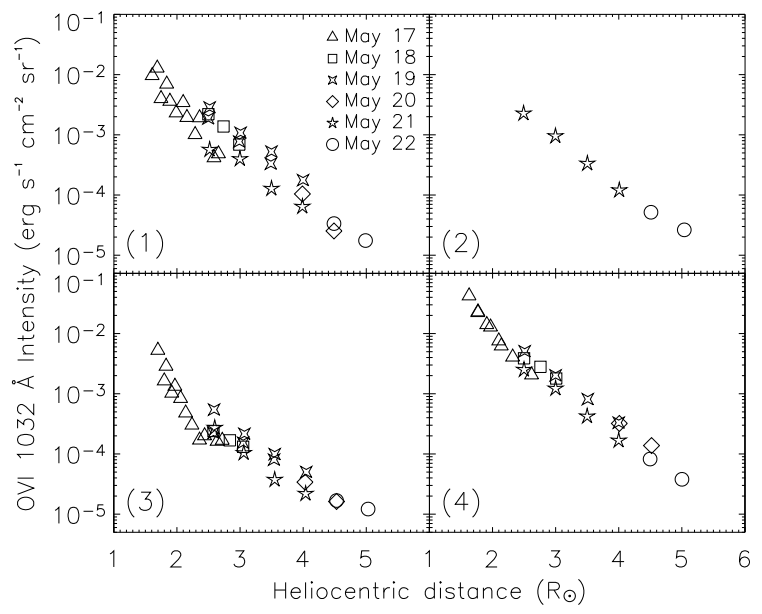

Fig. 2. O VI $1032 \AA$ A line-integrated intensities reported versus heliocentric distance along the southern (top-left panel) and northern (top-right panel) boundaries of the streamer, the out-of-streamer region (bottomleft panel) and the central region of the streamer (bottom-right panel). Different symbols refer to different observation days according to the labels reported top-left. The number in each panel denotes the corresponding observed region as represented in Fig. 1. The error bars, determined as described in the text, are not visible because they are smaller than the symbol size.

\section{Results and discussion}

\subsection{Behavior of the line intensities and profiles}

Figure 2 shows the total intensity of the O VI $1032 \AA$ line, as a function of heliocentric distance along the southern and the northern boundaries of the streamer, as well as in the region outside the streamer, adjacent to its southern border. The corresponding quantity observed in the central region of the streamer on the same days is also reported for comparison. The errors affecting the data points were computed as in Paper I: the corresponding $\pm \sigma$ values are plotted as error bars in the figure.

The total intensity radial profiles along the borders of the streamer (Fig. 2, top panels) fall off with height by at least two orders of magnitude in the examined heliocentric distance range. The data points referring to the northern boundary are higher than those corresponding to the southern one at any heliocentric distance (by a factor ranging from about 5 at $2.5 R_{\odot}$ to about 2 at $5.0 R_{\odot}$ ). In both cases the intensity values are systematically lower than those observed in the central region of the streamer (Fig. 2, bottom-right panel) on the same days by a factor of 3.5-4.

As for the intensity radial profile in the region adjacent to the southern border of the streamer (Fig. 2, bottom-left panel), it exhibits a decrease a little less than three orders of magnitude in the range of heliocentric distance considered. These intensity values are, on average, a factor of 3.5 lower than those measured along the streamer boundaries and by a little less than one order of magnitude lower than the corresponding data points obtained in the central region of the streamer during the same observational days. This can be ascribed to the lower density in the regions outside the streamer, as is evident in the LASCO C2 white-light images (see, e.g., Fig. 2 of Paper I).

The line-of-sight component of the O VI kinetic temperature relevant to the boundaries of the streamer (Fig. 3, top panels) shows the same increasing trend as measured along the central region of the observed structure (Fig. 3, bottom-right panel). The data points referring to the northern border are systematically

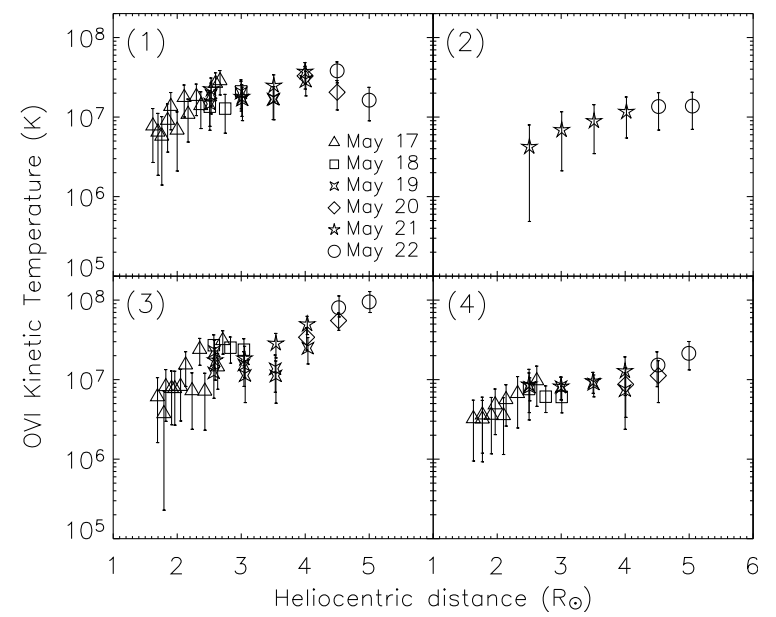

Fig. 3. Same as Fig. 2 for the O VI perpendicular (i.e. along the line of sight) kinetic temperature. See the text for a description of the error bar determination.

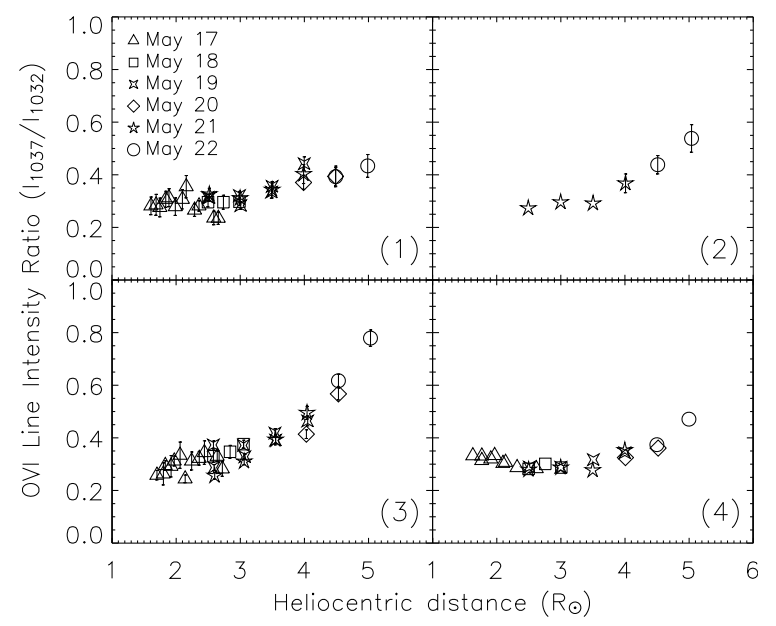

Fig. 4. Same as Fig. 2 for the O VI (1037 $\mathrm{A} / 1032 \AA)$ line ratios. The error bars, determined as described in the text, are plotted only if greater than the symbol size.

lower than those corresponding to the southern one, which, in turn, are 1.5-2 times higher than in the central region at all heliocentric distances.

The O VI kinetic temperature radial profile in the region adjacent to the southern border of the streamer (Fig. 3, bottomleft panel) is characterized by a steep increase from $\sim 4-7 \mathrm{MK}$ at $1.6 R_{\odot}$ to $\sim 100 \mathrm{MK}$ at $5.0 R_{\odot}$. The data points almost overlap with those referring to the borders of the streamer within $3.5 R_{\odot}$, while at greater heliocentric distances, the kinetic temperature in the out-of-streamer region is systematically higher. Moreover, the temperature values are several times higher than those inferred along the central region of the monitored structure on the same days, at all heights. The behavior of the O VI kinetic temperature radial profiles might suggest that the same heating mechanism acting inside the streamer is working along its boundaries, and even more efficiently in the region outside the streamer, probably due to the different magnetic configuration (open vs. closed) of this region (see, e.g. Frazin et al. 2003; Ventura et al. 2005).

The O VI intensity ratios relevant to both the southern and northern boundaries of the streamer (Fig. 4, top panels) range between 0.25 and 0.35 below $3 R_{\odot}$. At greater heliocentric distances, the observed ratios increase up to $0.45-0.5$. These data 


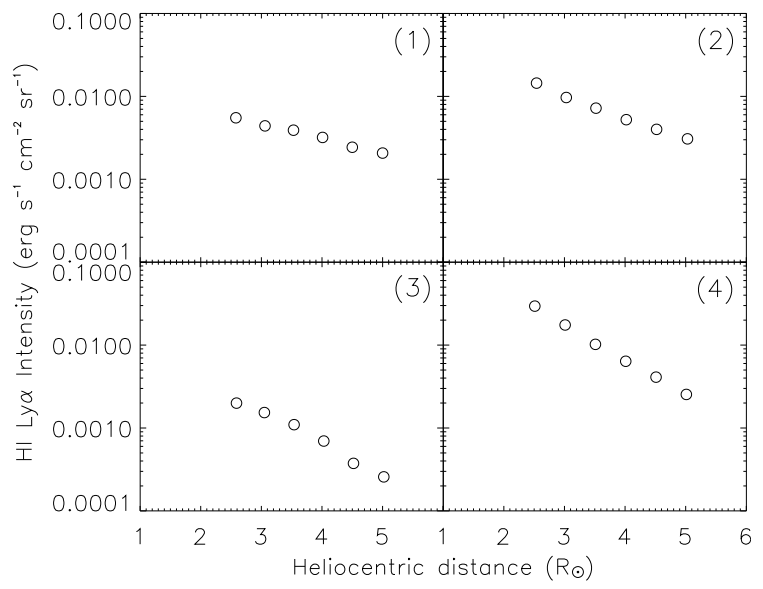

Fig. 5. H I Ly $\alpha$ line-integrated intensities versus heliocentric distance, as observed during the run of 2004 May 22-23 along the southern (topleft panel) and northern (top-right panel) boundaries of the streamer, the out-of-streamer region (bottom-left panel), and the central region of the streamer (bottom-right panel). The number in each panel denotes the corresponding observed region as represented in Fig. 1. The error bars, determined as described in the text, are not visible because they are smaller than the symbol size.

points are spread more with respect to the O VI intensity ratio profile obtained inside the streamer (Fig. 4, bottom-right panel) on the same days, although they are characterized by very similar values at all heights.

The most evident feature of the radial profile of the O VI line intensity ratio in the region adjacent to the southern boundary of the streamer (Fig. 4, bottom-left panel) is its quickly increasing trend, from about $0.25-0.35$ below $3.2 R_{\odot}$ to about 0.8 at $5.0 R_{\odot}$. While up to about $3.0 R_{\odot}$ these intensity ratios are very similar, within the uncertainties, to those referring to both the central region and the boundaries of the streamer, at greater heliocentric distances they are higher and higher, reaching a value nearly twice that derived in the southern border of the structure at $5.0 R_{\odot}$. The differences between in- and out-of-streamer ratios, increasing with height, probably reflect the progressively increasing efficiency of the Doppler dimming effect in the regions flanking the streamer, where the magnetic field lines open and the solar wind is expected to accelerate more and more with height (see, e.g., Noci et al. 1987).

Figure 5 shows the total intensity of the H I Ly $\alpha$ line as a function of the heliocentric distance along the two boundaries of the streamer (top panels) and in the region adjacent to the southern border (bottom-left panel). For comparison, the corresponding quantity referring to the central region of the streamer is also reported (bottom-right panel). The errors affecting the data points have been estimated as in the case of O VI lines. Due to the high rate of charge exchange between protons and neutral hydrogen in the corona up to about $10 R_{\odot}$, we can use the Ly $\alpha$ line widths as a proxy for the velocity distribution of protons along the line of sight (e.g., Withbroe et al. 1982; Allen et al. 1998; Vásquez et al. 2003).

The Ly $\alpha$ intensity radial profiles along the two boundaries exhibit the same decreasing trend in the examined interval of heliocentric distances. The data points relevant to the southern boundary are lower than those referring to the northern one by little more than a factor of two at $2.5 R_{\odot}$ and nearly 1.5 times at $5.0 R_{\odot}$. In both cases the intensity values are in general lower than those observed along the central region of the streamer with vanishing differences at greater heights. The intensity

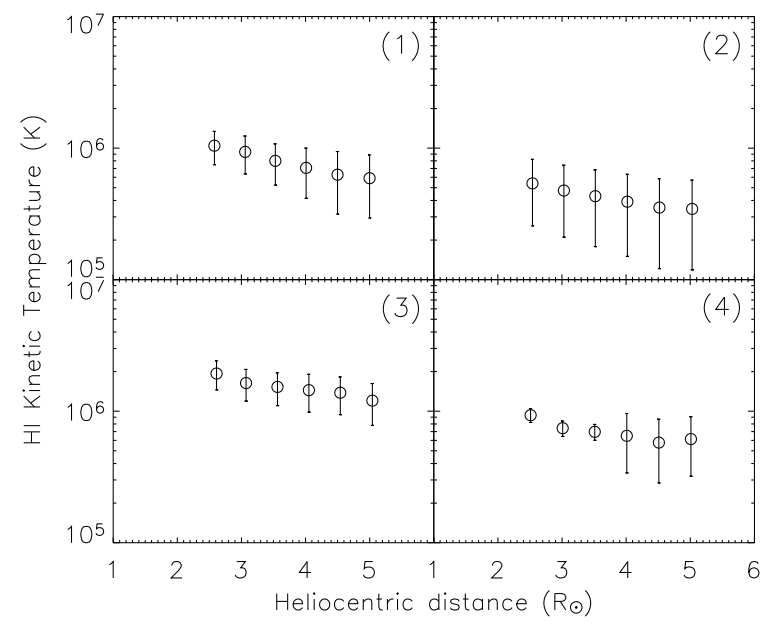

Fig. 6. Same as Fig. 5 for the H I perpendicular (i.e., along the line of sight) kinetic temperature. See the text for a description of the error bar determination.

distribution in the region outside the streamer exhibits the same decreasing trend, even if it is systematically lower, with values up to one order of magnitude less than in the borders. It is worth noting that the decrease in the Ly $\alpha$ intensity profiles is shallower than that of the O VI line observed on May 21 and 22 in the same range of heights, for all the regions considered here, because Ly $\alpha$ is resonantly scattered, while O VI $1032 \AA$ is collisionally excited.

Figure 6 reports the line-of-sight component of the Hi kinetic temperature as a function of the heliocentric distance for all the considered regions. The radial distribution of the H I kinetic temperature along the southern border of the streamer (top-left panel) exhibits a mild decrease from $1 \mathrm{MK}$ to about $0.6 \mathrm{MK}$, in the 2.5-5.0 $R_{\odot}$ range of heights. These temperature values are slightly higher than those observed in the region inside the streamer (bottom-right panel), while the values referring to the northern border (top-right panel) are on average about a factor of two lower at any heliocentric distance; however, such differences might not be significant, due to the error bars affecting the data points.

As for the out-of-streamer region (Fig. 6, bottom-left panel), the kinetic temperatures are more than a factor of two higher than those measured along the southern boundary of the structure, although they exhibit the same decreasing trend. These properties generally agree with the hypothesis of a heating mechanism that acts more efficiently in the regions surrounding the streamer, where an open magnetic configuration is expected. However, the main feature is the opposite behavior of the kinetic temperature of the $\mathrm{H}$ I atoms in comparison with the $\mathrm{O}$ VI ions. The characteristic decreasing trend of the former was also present in the data referring to the central region of the streamer (see Sect. 3.1 of Paper I).

\subsection{Derivation of the physical parameters}

The radial profiles of the electron density and temperature, kinetic temperature, and outflow velocity of the emitting ions along the boundaries of the streamer and in its adjacent region were derived from the empirical data presented in the previous subsection, using the same iterative approach as described in Sect. 3.2 of Paper I. To take possible variations in the physical parameters due to a temporal evolution of the observed structure into account (caused, for instance, by the solar rotation or 


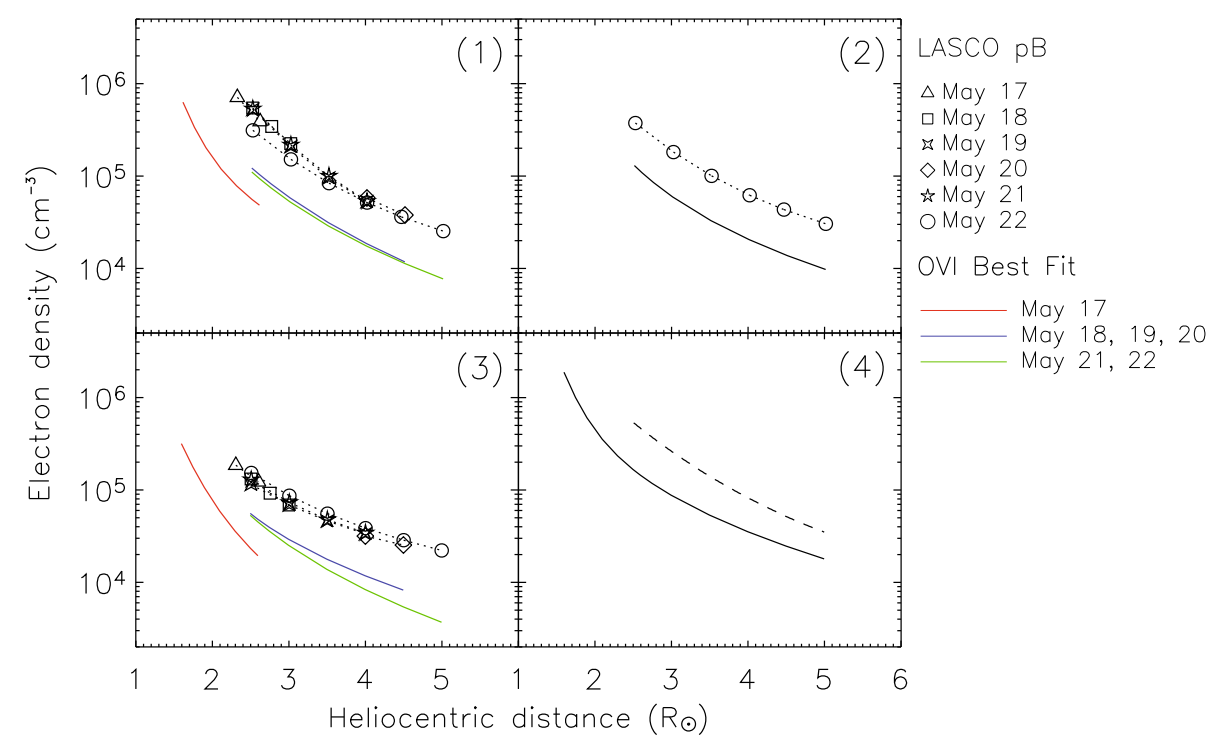

Fig. 7. Electron densities obtained from the inversion of LASCO C2 pB data (open points connected by dotted lines) and electron density radial profiles giving the best synthesis of O VI spectral data (solid color lines) derived along the southern (top-left panel) and northern (top-right panel) boundaries of the streamer, the out-of-streamer region (bottom-left panel) and the central region of the streamer (bottomright panel). Different symbols and colors refer to different days of observation according to the legend. The number in each panel denotes the corresponding observed region as represented in Fig. 1. changes in the global structure of the streamer), we grouped the $\mathrm{O}$ VI spectral data into three subsets, containing the observations collected on May 17, May 18, 19, and 20, and May 21, 22, respectively. Our choice was motivated by the line intensities relevant to May 18, 19, and 20 being systematically higher than those obtained during the other days. We then derived the plasma conditions for the three subsets separately.

Figure 7 reports the electron density radial profiles deduced from the inversion of the LASCO C2 pB data collected during the days of observation, together with those giving the best synthesis of the observed O VI emission line intensities, profiles, and intensity ratios, for both the streamer boundaries (top panels) and the out-of-streamer region (bottom-left panel). The density curves obtained in the case of the central region of the streamer (bottom-right panel, see also Paper I) are also shown for comparison purposes.

The different profiles deduced by the LASCO C2 pB data inversion during the various observation days exhibit a similar behavior, both along the boundaries and in the region adjacent to the streamer. They decrease by about one order of magnitude in the considered range of heliocentric distance. Moreover, the differences among the various curves are always within a factor of two in both cases. The density values along the boundaries of the streamer are slightly lower than those deduced from LASCO data within the central region of the structure, by a factor of 1.5 on average. The density values derived outside the streamer, conversely, are significantly lower than along the axis: by a factor of about 4 at $1.6 R_{\odot}$ and about two at $5.0 R_{\odot}$.

We used the electron density values relevant to May 22 to synthesize the H I Ly $\alpha$ line intensities and profiles, both along the streamer boundaries and in the adjacent region, getting good agreement, within the uncertainties, with the observed spectral data reported in Figs. 5 and 6 at all heights.

However, as in the case of the central region of the streamer, the density values obtained from LASCO C2 data are too high to consistently reproduce the observed O VI emission line intensities, profiles, and intensity ratios (see the detailed discussion of this issue in Paper I). The density profiles giving the best synthesis of the observed O VI spectral data are systematically lower, in general by a factor of 3.5 in the $2.5-5.0 R_{\odot}$ range of heliocentric distance. This difference is larger than the estimated uncertainties in the determination of the electron density, which are below a factor of two (cf. Hayes et al. 2001; Strachan et al. 2002).
For a possible explanation of this apparent discrepancy, we refer the reader to the detailed discussion reported in Paper I, considering that the observed O VI emission probably originated in a narrow structure away from the plane of the sky, contributing little brightness compared to the background corona, whereas the $\operatorname{Ly} \alpha$, and most of white-light radiation, could originate in a wider and denser region along the line of sight.

The curves obtained by matching the $\mathrm{O}$ VI observations and relevant to the streamer boundaries (Fig. 7, top panels) exhibit systematically lower density values than those corresponding to the central region of the streamer (Fig. 7, bottom-right panel), by about a factor of 3 in the 1.6-2.5 $R_{\odot}$ range of heliocentric distances (i.e., for observations collected on May 17) and by about a factor of two at greater heights. The density profile relevant to the northern boundary (Fig. 7, top-right panel) overlaps that corresponding to the southern one practically at all heliocentric distance except above $4.0 R_{\odot}$, where it is slightly higher. The outof-streamer density profiles (Fig. 7, bottom-left panel) are even lower than those relevant to the central region of the streamer by about a factor of 4-5. The profiles giving the best synthesis of O VI spectral data also show significant variations during the different observational days, while the curves relevant to May 18, 19, 20 and May 21, 22 are almost consistent. The density values fitting the observations collected on May 17 are systematically lower than those corresponding to the other days, by about a factor of 2.5 at $2.6 R_{\odot}$ along the borders of the streamer and by about a factor of 3 at the same height in its adjacent region. This variability reflects the fluctuations found in the O VI emission line intensity radial profiles on the same days (see Fig. 2) and can be reasonably ascribed to both the changing shape and aspect of the streamer and surrounding regions during the observational week, although some effect of the CME which occurred on May 18 in the northward region of the examined structure cannot be ruled out.

The electron temperature $\left(T_{\mathrm{e}}\right)$ radial profiles along the borders of the streamer and in its adjacent region were derived from the corresponding density profiles by the same technique adopted in Paper I and described by Gibson et al. (1999), which works under the assumption that the coronal plasma is in radial hydrostatic equilibrium. This means that there are no significant dynamic forces and that thermal pressure is balanced by gravity. This hypothesis could be considered fairly valid for the plasma located across the boundaries of the examined streamer, since 


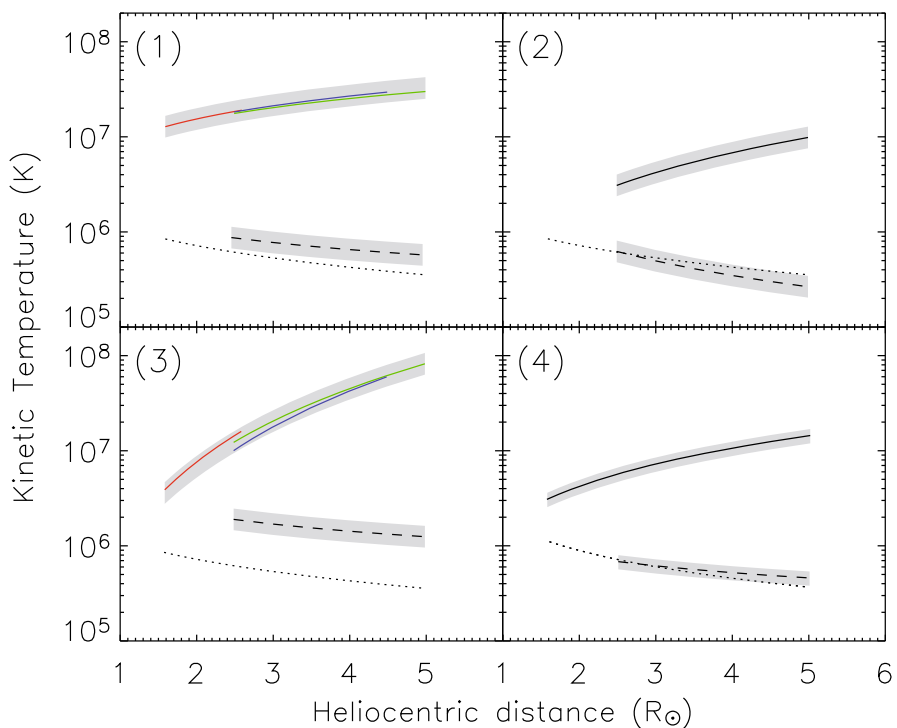

the velocities reached by the $\mathrm{O}$ VI ions and the hydrogen atoms at $5.0 R_{\odot}$ in these regions are about $120 \mathrm{~km} \mathrm{~s}^{-1}$, so well below the thermal velocities of electrons corresponding to the resulting temperatures at all heights. In the out-of-streamer regions, however, the assumption of radial hydrostatic equilibrium still holds only at low heliocentric distances, while at greater heights the high outflow velocity of particles determined in this study cannot be considered as a minor term in the force balance (see below). In this case, therefore, the values of electron temperature deduced from the electron density, according to Gibson et al. (1999), are only a first approximation estimate.

The electron temperature profiles resulting from the different density profiles reported in Fig. 7 do not exhibit significant differences between both the streamer boundaries and the region adjacent to the southern border of the structure. Therefore we averaged the various electron temperature profiles and obtained a common curve for these three regions. This curve, reported in Fig. 8 (top and bottom-left panels), is less than $30 \%$ lower than the profile derived in the region inside the streamer (bottom-right panel, see also Paper I).

Figure 8 also shows the empirical radial profiles of the component of the kinetic temperature in the direction perpendicular to the magnetic field $\left(T_{\perp}\right)$ deduced for $\mathrm{O}$ VI ions and $\mathrm{HI}$ atoms (protons) along the streamer boundaries and in the outof-streamer region. The average uncertainty ranges affecting the radial profiles, reported in the figure, were derived in terms of the lowest and highest temperature values that allow a consistent reproduction of the observed line intensities, profiles, and $\mathrm{O}$ VI intensity ratios, within the observational uncertainties.

The O VI perpendicular kinetic temperature along the southern border of the streamer (Fig. 8, top-left panel) ranges from about $10 \mathrm{MK}$ at $1.6 R_{\odot}$ to about $30 \mathrm{MK}$ at $5.0 R_{\odot}$, with values that are systematically higher than those derived along the central region of the streamer (Fig. 8, bottom-right panel), by a factor of about 3.5 at $1.6 R_{\odot}$ and about 2 at $5.0 R_{\odot}$. The temperature profile along the northern boundary (Fig. 8, top-right panel), conversely, exhibits a similar increasing behavior than that obtained along the streamer axis, although it is 1.5 times lower on average. In the streamer adjacent region (Fig. 8, bottom-left panel) the temperature radial profile abruptly increases from about $3.5 \mathrm{MK}$ at $1.6 R_{\odot}$ to nearly $100 \mathrm{MK}$ at $5.0 R_{\odot}$, with values much higher (up to nearly one order of magnitude) than those obtained inside the

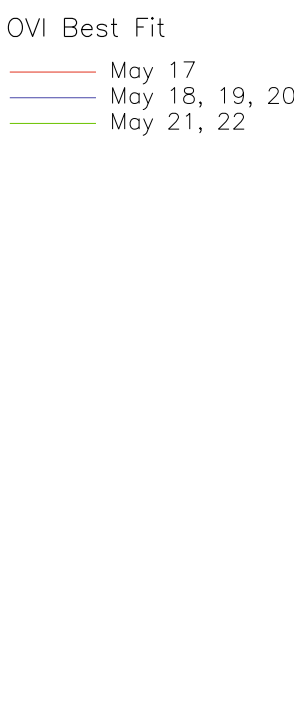

Fig. 8. Radial profiles of the O VI (solid color lines) and $\mathrm{HI}$ (dashed black lines) kinetic perpendicular temperature $\left(T_{\perp}\right)$ empirically derived by iteratively reproducing as closely as possible the data reported in Figs. 3 and 6, respectively. They are shown versus heliocentric distance along the southern (top-left panel) and northern (top-right panel) boundaries of the streamer, the out-of-streamer region (bottom-left panel), and the central region of the streamer (bottom-right panel). Different colors refer to different observational days according to the legend. Also reported is the averaged radial profile of the electron temperature $T_{\mathrm{e}}$ (dotted line), determined as described in the text. The number in each panel denotes the corresponding observed region as represented in Fig. 1. The shaded bands represent the average ranges of uncertainties affecting the temperature curves.

streamer. Note that the curves relevant to different days exhibit no significant variations.

The radial profiles of the H I perpendicular kinetic temperature are all much lower (up to about two orders of magnitude) than the corresponding O VI profile. Along both the boundaries of the streamer, they show a slight decrease in the 2.5-5.0 $R_{\odot}$ interval of heliocentric distance and do not diverge from the corresponding curve relevant to the central region of the streamer by more than $50 \%$. Differences of the same order of magnitude or even lower can be noticed when comparing the electron temperature profile with the $\mathrm{HI} T_{\perp}$ curves along both the streamer borders. Therefore it seems reasonable that the hydrogen atoms (and then protons) in these regions are nearly in thermal equilibrium with electrons, as well as along the central region of the streamer (see Paper I). This is not the case in the region adjacent to the southern border of the structure, where the radial profile of the HI perpendicular kinetic temperature is higher than the corresponding electron temperature curve, by a factor of 3 on average. Note, also, that the Hi kinetic temperature is higher outside the streamer than in its central region by the same factor.

As for the components of the O VI and Hi kinetic temperatures parallel to the magnetic field lines $\left(T_{\|}\right)$, not reported in Fig. 8, the best synthesis of the empirical data set relevant to the streamer borders was obtained considering slightly anisotropic conditions with $T_{\|}=T_{\perp} / 1.3$, according to what has already been found in the analysis of the central region of the streamer (see Paper I and Frazin et al. 2003). Outside the streamer we achieved the best synthesis of the H I spectral data with the same level of anisotropy in the kinetic temperature. In the case of O VI a higher anisotropy $\left(T_{\|}=T_{\perp} / 2.0\right)$ is required for the best fit to the observations.

The empirical radial profiles of the O VI and H I outflow velocity along the boundaries of the streamer and in its adjacent region are reported in Fig. 9. They were obtained by adopting the same procedure as described in Sect. 3.2 of Paper I. The corresponding quantity along the central region of the streamer structure is also reported for comparison. The error bars of $\pm 25 \mathrm{~km} \mathrm{~s}^{-1}$, on average, affecting each point were deduced as in the case of the kinetic temperature uncertainties.

The outflow velocity values derived for both O VI ions and neutral hydrogen atoms along the southern boundary of the streamer (Fig. 9, top-left panel) are generally consistent, within the uncertainties, with those obtained for the northern one 


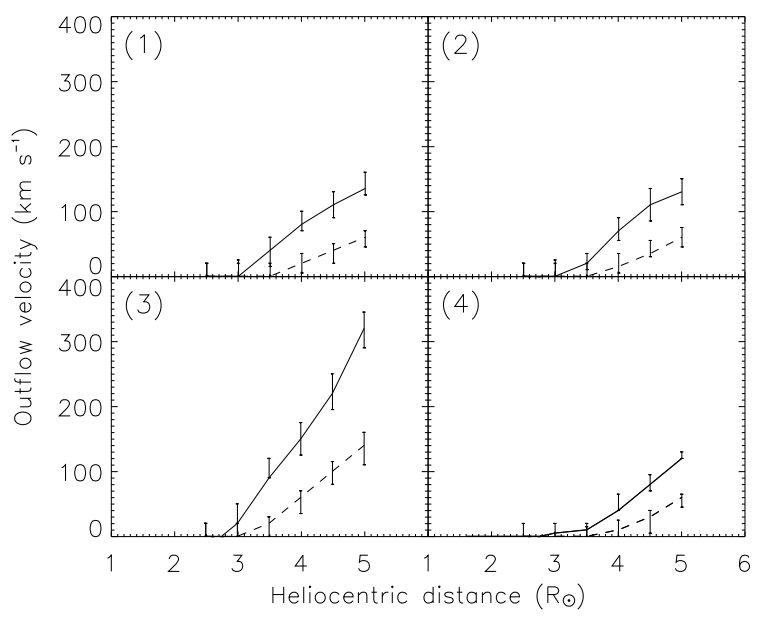

Fig. 9. Radial profiles of the O VI (solid lines) and H I (dashed lines) outflow velocity derived along the southern (top-left panel) and northern (top-right panel) boundaries of the streamer, the out-of-streamer region (bottom-left panel) and the central region of the streamer (bottom-right panel). The number in each panel denotes the corresponding observed region as represented in Fig. 1.

(Fig. 9, top-right panel). Evidence of significant deviation from static conditions only appears above $3.0 R_{\odot}$ for both of them. The O VI outflow velocity along the borders of the streamer is in general greater than in the central region of the structure (Fig. 9, bottom-right panel) by about a factor of 1.5-2, reaching values around $135 \mathrm{~km} \mathrm{~s}^{-1}$ at $5.0 R_{\odot}$. Moreover, its radial profile is steeper along the boundaries, below $4.0 R_{\odot}$. No significant differences have been found for the H I outflow velocity between the radial profiles derived along the boundaries of the streamer and in its central region; moreover, the respective curves reach the same value of $60 \mathrm{~km} \mathrm{~s}^{-1}$ at $5.0 R_{\odot}$.

The out-of-streamer scenario (see Fig. 9, bottom-left panel) is substantially different, even if static conditions still appear present below $3.0 R_{\odot}$. The $\mathrm{O}$ VI outflow velocity profile abruptly increases above $3.0 R_{\odot}$, from values around $20 \mathrm{~km} \mathrm{~s}^{-1}$ to $320 \mathrm{~km} \mathrm{~s}^{-1}$ at $5.0 R_{\odot}$. This speed value is nearly 3 times higher than obtained at the same height in the central region of the streamer. The H I outflow velocity increases more gradually than does that of $\mathrm{O}$ VI, reaching a speed of about $150 \mathrm{~km} \mathrm{~s}^{-1}$ at $5.0 R_{\odot}$, that is, 2.5 times higher than derived in the central region of the streamer at the same height.

It is worth noticing that the outflow velocity of coronal O VI ions at lower heights might be underestimated in this analysis: we adopted the quiet Sun O VI line disc intensities reported in Vernazza \& Reeves (1978) to perform the synthesis of $\mathrm{O}$ VI emission in the examined regions, obtaining a very consistent agreement with the observations. However, a non-negligible $\mathrm{O}$ VI outflow velocity below $3.0 R_{\odot}$ could result from the analysis of the observed line intensity ratio, when the possible influence of the active region sunspots (present on the solar disc during the week of observation, see Fig. 4 in Paper I) on the intensity of the radiative scattering component of the coronal $\mathrm{O}$ VI lines is also considered, as discussed by Morgan \& Habbal (2005).

We finally note that, in all the examined regions, the O VI ions appear to flow significantly faster (2-3 times) than the $\mathrm{HI}$ ones. This, together with the considerably higher O VI kinetic temperatures found in the examined regions, confirms the possibility of energy deposition and plasma heating and acceleration through the absorption of Alfvén waves at ion cyclotron frequency (e.g., Li et al. 1997, 1999; Tu \& Marsch 1997;
Cranmer et al, 1999a, 1999b; Hu \& Habbal, 1999), both along the boundaries of the streamer structure and immediately outside it.

\section{Summary and conclusions}

Through the analysis of the intensity and profile of the H I Ly $\alpha$ and $\mathrm{O}$ VI resonant doublet lines, we empirically determined the plasma parameters at different heliocentric distances along the boundaries and in a region adjacent to a mid-latitude streamer, observed through a week in May 2004, during the declining phase of the solar activity cycle 23 .

The electron density values deduced by both the LASCO C2 $\mathrm{pB}$ data and $\mathrm{O}$ VI spectral data are lower than the corresponding ones inferred inside the streamer by a factor of 2-3 along the borders and by a factor of 3-5 in the out-of-streamer region. This behavior has already been described in the literature: in their analysis of several mid-latitude streamers observed in 1997 and 2000 (i.e., during the minimum and the maximum of solar activity), Ventura et al. (2005) found that the out-of-streamer regions are characterized by densities up to one order of magnitude lower than those relevant to the regions inside the streamers; similar results have also been obtained by Antonucci et al. (2005), who analyzed a collection of equatorial streamers at solar minimum.

The electron temperature radial profiles deduced both along the boundaries of the streamer and in its adjacent region exhibit the same decreasing trend as inferred along the central region of the structure, with only slightly lower values. These modest differences are consistent with the results of Antonucci et al. (2005), who derived a radial profile of $T_{\mathrm{e}}$ for regions adjacent to streamers and compared it to the profile obtained by Gibson et al. (1999) along the streamer's central regions. The component of the O VI kinetic temperature in the direction perpendicular to the magnetic field is characterized by a steep increase with height, both along the boundaries of the streamer and in its surroundings, reaching values considerably higher than in the central region of the streamer. A similar trend characterizes the results reported in Antonucci et al. (2005), Spadaro et al. (2005) and Ventura et al. (2005), who found significantly higher $\mathrm{O}$ VI temperatures in the regions adjacent to the observed streamers. However, these authors did not notice appreciable differences between the in- and out-of-streamer radial profiles of the H I kinetic temperature, whereas we found on average a factor of 3 higher temperatures outside the streamer. Conversely, Uzzo et al. (2007) found H I temperatures up to a factor of about 2 higher inside the mid-latitude streamers considered in their analysis, compared to outside, between 1.75 and $5.0 R_{\odot}$. In one case only, at $5.0 R_{\odot}$, they noticed a temperature value slightly lower in the central region of a streamer with respect to its surroundings.

Evidence of a non-negligible outflow velocity of O VI and H I ions appears at 3.0-3.5 $R_{\odot}$, both along the borders of the streamer and in the out-of-streamer region, while static plasma conditions hold at lower heights. The outflow velocity of the $\mathrm{O}$ VI ions significantly increases in the considered range of heliocentric distances, while the hydrogen atoms appear to accelerate more gradually.

The $\mathrm{O}$ VI outflow velocity behavior determined here is generally in good agreement with that inferred by Habbal et al. (1997) in the first study of the transition from fast to slow wind across a streamer using UVCS measurements. These authors reported O VI speeds of about $94 \mathrm{~km} \mathrm{~s}^{-1}$ at $4.5 R_{\odot}$, in the central region of the examined streamers, while, at increasing distances from the streamer axis, they found the same value closer to the 
Sun, namely at 3.5-4.0 $R_{\odot}$ and $2.5-3.0 R_{\odot}$ at $P A= \pm 10^{\circ}$, and $P A= \pm 20^{\circ}$ from the streamer axis, respectively. This is also compatible with the latitudinal gradient in O VI wind speed occurring very close to the streamer borders in our data.

The O VI velocity derived outside the streamer, examined here at $3.5 R_{\odot}$, is consistent, within the uncertainties, with the value $\left(\sim 115 \mathrm{~km} \mathrm{~s}^{-1}\right)$ reported by Antonucci et al. (2005) at the same height in regions lying in the immediate vicinity of streamers. However, these authors found significant velocities even below $3.0 R_{\odot}$ : already at $1.8 R_{\odot}$, for instance, they got a value of $\sim 90 \mathrm{~km} \mathrm{~s}^{-1}$, that is comparable with those derived by Cranmer et al. (1999b) at the same height in the core of polar coronal holes during the minimum of solar activity. These differences could probably be ascribed to the fact that the out-of-streamer regions observed by Antonucci et al. (2005) are nearly 1.5 times wider than those considered in our analysis and on average $30^{\circ}$ far from the respective streamer axis, while in our case the maximum separation is about $20^{\circ}$, so the out-of-streamer strips examined by Antonucci et al. (2005) could intersect wider parts of the large coronal holes located around the solar poles during activity minimum, where the acceleration of the wind could take place already at low heliocentric distances, resulting in greater outflow velocities. On the other hand, we cannot exclude that the $\mathrm{O}$ VI ion speed values deduced from our analysis could be underestimated below $3.0 R_{\odot}$, as described at the end of Sect. 3.2.

An interesting feature is that the enhancements in the perpendicular kinetic temperature of O VI and, to a lesser extent, of H I already appear to increase at heliocentric distances below $3.0 R_{\odot}$. Probably, the same mechanism responsible for the plasma heating at low heights also gradually accelerates the wind particles, which reach detectable velocities only at larger heliocentric distances. Similar differences between the kinetic and dynamical plasma responses were also detected by Spadaro et al. (2005), in their analysis of plasma parameters inside and outside mid-latitude streamers at solar minimum, and by Ventura et al. (2005), whose observed O VI line intensity ratios are in excellent agreement with those reported in this work.

The H I and O VI outflow velocities in the region adjacent to the southern border of the streamer are significantly lower (by a factor of 4-5) than those characterizing the fast wind flowing in polar coronal holes (see, e.g., Kohl et al. 1998; Cranmer et al. 1999b; Antonucci et al. 2004; Telloni et al. 2007). This clearly indicates that the plasma located immediately outside the streamer is in a regime of slow flow, even if its velocity is higher than along both the boundaries and inside the streamer above the cusp, i.e., the region where the transition between closed and open magnetic field lines takes place and the heliospheric current-sheet forms.

Hence we can conclude, in agreement with Antonucci et al. (2005), that two components of slow wind probably form in the observed regions. One is flowing along the magnetic flux tube formed by the open field lines delimiting the streamer structure, with only slightly lower O VI velocities at 5.0 $R_{\odot}$ than the slow wind asymptotic heliospheric value of approximately $400 \mathrm{~km} \mathrm{~s}^{-1}$. The other one originates just above the streamer cusp, in our case located between 3.5 and $4.0 R_{\odot}$, and flows with significantly slower speed than in the regions flanking the structure. Our investigation showed for the first time that this bi-modal behavior also occurs for the H I velocities in the examined regions. The differences between these two slow-wind components suggest that they are probably of different nature, and the particle flux observed above the streamer cusp cannot be considered simply as the merging of the wind flowing in the regions surrounding the streamer structure (see, e.g., Noci et al. 1997; Raymond et al. 1997; Antonucci et al. 2005).

Acknowledgements. The authors wish to thank Y.-K. Ko for her assistance during UVCS/SOHO observations carried out at NASA GSFC, and R. Suleiman for his help in the preliminary reduction of the UVCS raw data. They also thank the referee for providing very useful comments. This work was supported in part by the Agenzia Spaziale Italiana (contract I/035/05/0). Financial support by the European Commission through the SOLAIRE Network (MTRN-CT-2006035484) is also gratefully acknowledged. The work of E. Landi is supported by NASA grants.

\section{References}

Abbo, L., \& Antonucci, E. 2002, in SOHO 11 Symp., From Solar Minimum to Max: Half a Solar Cycle with SOHO, ed. A. Wilson (Noordiwijk: ESA), ESA-SP, 508, 477

Allen, L. A., Habbal, S. R., \& Hu, Y. Q. 1998, JGR, 103, 6551

Antonucci, E., Dodero, M. A., Giordano, S., Krishnakumar, V., \& Noci, G. 2004, A\&A, 416, 749

Antonucci, E., Abbo, L., \& Dodero, M. A. 2005, A\&A, 435, 699

Antonucci, E., Abbo, L., \& Telloni, D. 2006, ApJ, 643, 1239

Brueckner, G. E., Howard, R. A., Koomen, M. J., et al. 1995, Sol. Phys., 162, 357

Cranmer, S. R., Field, G. B., \& Kohl, J. L. 1999a, ApJ, 518, 937

Cranmer, S. R., Kohl, J. L., Noci, G., et al. 1999b, ApJ, 511, 481

Domingo, V., Fleck, B., \& Poland, A. I. 1995, Sol. Phys., 162, 1

Frazin, R. A., Cranmer, S. R., \& Kohl, J. L. 2003, ApJ, 597, 1145

Gibson, S. E., Fludra, A., Bagenal, F., et al. 1999, JGR, 104, 9691

Gouttebroze, P., Lemaire, P., Vial, J. C., \& Artzner, G. 1978, ApJ, 225, 655

Habbal, S. R., Woo, R., Fineschi, S. et al. 1997, ApJ, 489, L103

Hayes, A. P., Vourlidas, A., \& Howard, R. A. 2001, ApJ, 548, 1081

Hu, Y. Q., \& Habbal, S. R. 1999, JGR, 104, 17045

Kohl, J. L., Esser, R., Gardner, L. D., et al. 1995, Sol. Phys., 162, 313

Kohl, J. L., Noci, G., Antonucci, E., et al. 1997, Sol. Phys., 175, 613

Kohl, J. L., Noci, G., Antonucci, E., et al. 1998, ApJ, 501, L127

Morgan, H., \& Habbal, S. R. 2005, ApJ, 630, L189

Noci G., Kohl, J. L., \& Withbroe, G. L. 1987, ApJ, 315, 706

Noci, G., Kohl, J. L., Antonucci, E., et al. 1997, in Fifth SOHO Workshop, The Corona and Solar Wind near Minimum Activity, ed. A. Wilson (ESA-SP 404; Noordwijk: ESA), 75

Raymond, J. C., Kohl, J. L., Noci, G., et al. 1997, Sol. Phys., 175, 645

Spadaro, D., Ventura, R., Cimino, G., \& Romoli, M. 2005, A\&A, 429, 353

Spadaro, D., Susino, R., Ventura, R., Vourlidas, A., \& Landi, E. 2007, A\&A, 475, 707 (Paper I)

Strachan, L., Suleiman, R., Panasyuk, A. V., Biesecker, D. A., \& Kohl, J. L. 2002, ApJ, 571, 1008

Telloni, D., Antonucci, E., \& Dodero, M. A. 2007, A\&A, 472, 299

Uzzo, M., Ko, Y.-K., Raymond, J. C., Wurz, P., \& Ipavich, F. M. 2003, ApJ, 585, 1062

Uzzo, M., Strachan, L., Vourlidas, A., Ko, Y.-K., \& Raymond, J. C. 2006, ApJ, 645,720

Uzzo, M., Strachan, L., \& Vourlidas, A. 2007, ApJ, 671, 912

Vásquez, A. M., van Ballegooijen, A. A., \& Raymond, J. C. 2003, ApJ, 598, 1361

Ventura, R., Spadaro, D., Cimino, G., \& Romoli, M. 2005, A\&A, 430, 701

Vernazza, J. E., \& Reeves, E. M. 1978, ApJS, 37, 485

Wang, A. H., Wu, S. T., Suess, S. T., Poletto, G. 1998, JGR 103, 1913

Woo, R., \& Martin, J. 1997, GRL, 24, 2535

Wilhelm, K., Curdt, W., Marsch, E., et al. 1995, Sol. Phys.,162, 189

Withbroe, G. L., Kohl, J. L., Weiser, H., \& Munro, R. H. 1982, Space Sci. Rev., 33,17

Woods, T. N., Tobiska, W. K., Rottman, G. J., \& Worden, J. R. 2000, JGR, 105, 27195

Zangrilli, L., Nicolosi, P., Poletto, G., et al. 1999, A\&A, 342, 592 